\title{
Wheat and maize miRNAs are potential regulators of human genes expression
}

\author{
Aizhan Kazievna Rakhmetullina \\ SRI of biology and biotechnology \\ problems, Al-Farabi Kazakh National \\ University Almaty, Kazakhstan \\ zhanullina1994@gmail.com
}

\author{
Anatoliy Timofeevich Ivashchenko \\ SRI of biology and biotechnology \\ problems, Al-Farabi Kazakh National \\ University Almaty, Kazakhstan \\ a.iavashchenko@gmail.com
}

\author{
Anna Yurevna Pyrkova \\ SRI of biology and biotechnology \\ problems, Al-Farabi Kazakh National \\ University Almaty, Kazakhstan \\ anna.pyrkova@kaznu.kz
}

\begin{abstract}
With food, a huge variety of biological material gets into the human digestive tract, which the body uses for life support. The variety of food material entering the gastrointestinal tract, especially at the molecular level, cannot be distinguished from endogenous metabolites and these exogenous compounds can significantly alter the body's metabolism. Such compounds include plant miRNAs, which are indistinguishable from endogenous human miRNAs in physicochemical properties. It is necessary to clarify the degree of influence of exogenous plant miRNAs on the expression of human genes, since it is not known in advance what consequences can occur when plant miRNAs enters the human body. A huge amount of research does not allow experiments with all human genes and all plant miRNAs, so we have studied the effect of wheat and maize miRNAs on human genes using computer methods. As a result of studying the binding of $\mathbf{1 2 5}$ tae-miRNAs and 325 zma-miRNAs to mRNAs of 17508 human genes it was revealed that 158 genes were targets for $\mathbf{5 2}$ taemiRNAs and 51 genes for 11 zma-miRNAs. Binding sites in the mRNA of human genes were located in 5'UTR, CDS, 3'UTR.
\end{abstract}

Keywords - miRNA, mRNA, binding site, gene regulation, plant, wheat, maize, human

\section{Introduction}

Recently, publications have appeared about the ingestion of exogenous miRNAs into the human body. Plant miRNAs that enter the human body along with food are able to penetrate the tissues and affect some metabolic pathways. These extracellular miRNAs are stable and involved in intercellular interactions [1]. Wheat and maize are the most actively cultivated crops worldwide. It is imperative to learn how plant miRNAs can affect the human body and how these miRNAs can be useful or harmful. The MirTarget program used by us with high efficiency determines the quantitative characteristics of the interaction of plant miRNAs with human mRNAs.

\section{Materials and metods}

Nucleotide sequences of the mRNAs of human genes were obtained from NCBI (http://www.ncbi.nlm.nih.gov). Nucleotide sequences of wheat and maize miRNAs were downloaded from miRBase v.22 (http://www.mirbase.org/). The miRNA binding sites in mRNA of 17508 human genes were predicted using the MirTarget program, which defines the free energy of interaction miRNA and mRNA $(\Delta G$, $\mathrm{kJ} / \mathrm{mole}$ ), as well as the localization of binding sites and schemes of nucleotide interactions between miRNAs and mRNAs $[2,3]$. The ratio of $\Delta \mathrm{G} / \Delta \mathrm{Gm}(\%)$ was determined for each binding site, where $\Delta \mathrm{Gm}$ is equal to the free energy binding of miRNA with its full complementary nucleotide sequence.

\section{Results and discussion}

We found that among 325 miRNAs of Z. mays only 11 miRNAs were able to bind to mRNA of human genes. These miRNAs interacted with mRNA of 51 different genes. The zma-miRNA binding sites in the mRNA of human genes were located in 5'UTR, CDS, 3'UTR. The largest number of target genes had miR529-3p. The miR529-3p interacted with mRNA of ATP6V0A4, BZRAP1, CHD2, CNGA2, GRM4, HCLS1, HIVEP 2, IGFN1, LMO7, LRRC73, LRTOMT, LTB4R, MAP7, MLL, NUDC, PDE4B, POMC, SOX9, TCEB3, TMEM181 genes with the value of $\Delta \mathrm{G} / \Delta \mathrm{Gm}$ from $91 \%$ to $94 \%$. The miRNA binding sites in mRNA of 13 genes were located in CDS, four sites in 5'UTR and 3'UTR. The miR162-3p and miR11969-5p had binding sites in the mRNA of four and six target genes, respectively. The binding sites of these miRNAs were located only in CDS and $3^{\prime} \mathrm{UTR}$. The rest miRNAs (miR827-3p, miR529-5p, miR162-5p, miR1432-5p, miR11970-5p, and miR11969-3p) had only one target genes, with the value of $\Delta \mathrm{G} / \Delta \mathrm{Gm}$ more than $88 \%$. In the group of 11 miRNAs, there were four miR-3p/miR-5p pairs that belong to the same pre-miRNA (miR529-3p/5p miR482-3p/5p, $\operatorname{miR} 162-3 p / 5 p \operatorname{miR} 11969-3 p / 5 p)$. The characteristics of the binding sites for miR482-5p and miR482-3p were found for eight and nine mRNA target genes, respectively. Some of the above mentioned 17 genes involved in the development of diabetic retinopathy $(\mathrm{ROBO} 4)$ [4], papillary thyroid carcinoma (CDKN1C) [5], myeloid leukemia (FAM168A) [6], hypogonadotropic hypogonadism (FGF17) [7], pancreatic and gastric cancers (SLC44A4) [8], breast cancer (ATP2A3) [9], lung cancer (LARPI) [10]. The binding sites of these miRNAs were located at 5'UTR, CDS and 3'UTR, and the free binding energy was a $-96 \mathrm{~kJ} / \mathrm{mole}$ and $-98 \mathrm{~kJ} / \mathrm{mole}$.

As a result of the studies, binding sites were established between the wheat miRNAs and the mRNA of human genes involved in the development of various diseases. Binding sites were found for 52 miRNAs with mRNAs of 158 genes with a $\Delta \mathrm{G} / \Delta \mathrm{Gm}$ value of $88 \%$ or more. The binding sites of the studied miRNAs were located at 5'UTR (9\%), CDS (63\%) and 3'UTR (28\%) mRNA. The highest number of binding sites with high affinity was detected in CDS and 3'UTR mRNA of target genes. Data analysis showed that genes $A D A R B 2$, ADCY6, ALPK3, ANO4, BMS2, EDEM1, ERICH1, FNIP2, KIRREL3, NGB, PAQR6, TJP3,RICH1, RLBP1, SMARCC2, $U B E 2 K, U N G$ were targets for miR408-3p, with free energy from $-108 \mathrm{~kJ} /$ mole to $-113 \mathrm{~kJ} / \mathrm{mole}$ and $\Delta \mathrm{G} / \Delta \mathrm{Gm}$ value from $91 \%$ to $95 \%$. Therefore, miR408-3p can regulate a significant number of genes and further study is required. The miR105215p, miR1118-5p, miR1124-3p, miR1129-5p, miR1134-3p, miR1138-3p, miR164-5p, miR5086-5p, miR9652-5p, miR9655-3p, miR9661-5p, miR9773-3p, miR9780-3p, miR9781-3p bound to mRNA of four or more target genes. The functions of the identified target genes were diverse. 
The figure shows the interaction schemes of tae-mir4083p, zma-miR529-3p, and zma-miR482-3p with different regions of mRNA (5'UTR, CDS, 3'UTR) of human genes. The interactions between miRNA and mRNA show the role of noncanonical G-U and A-C pairs in increasing the free energy of interaction between miRNA and mRNA.

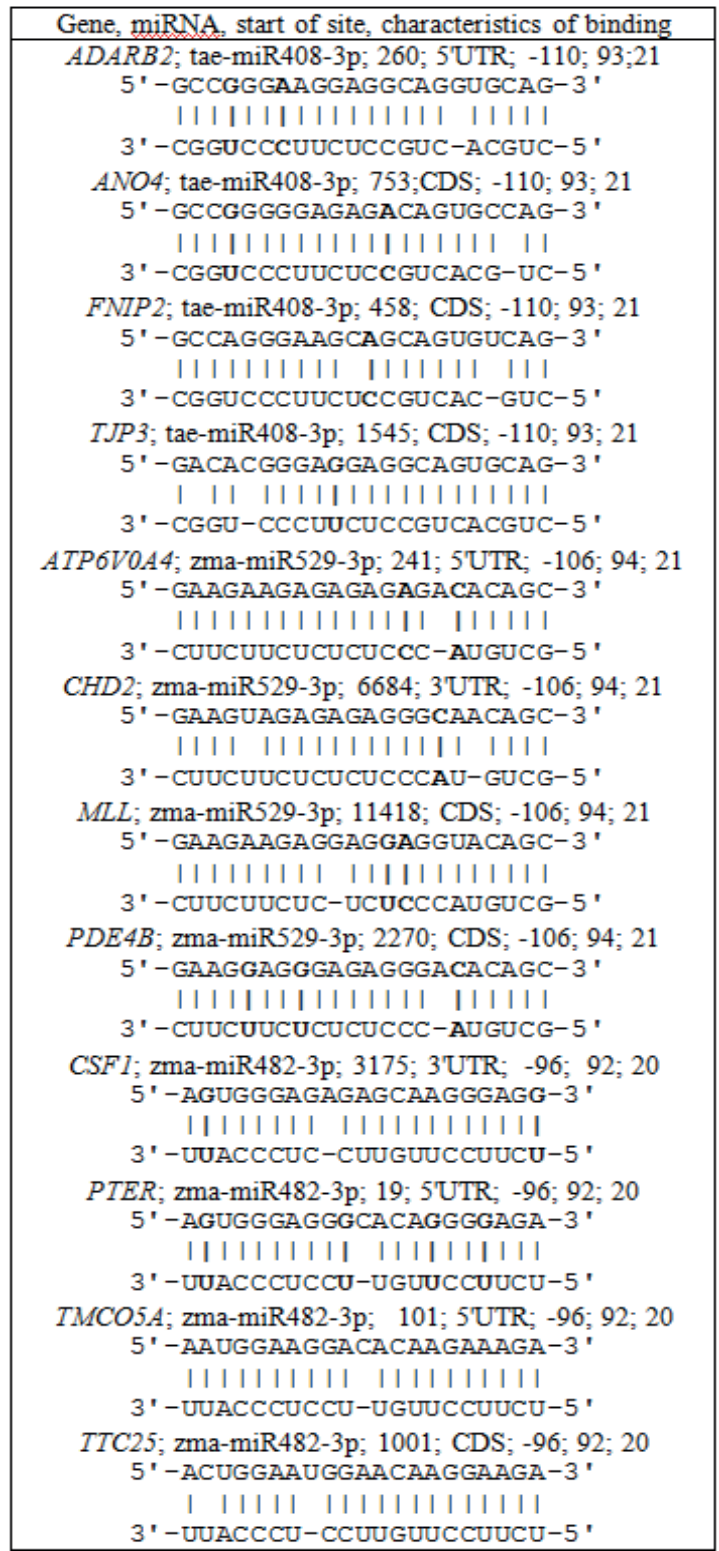

Fig. 1. - Schemes of the interaction of tae-miRNA and zma-miRNA with mRNA human genes

Note: The upper and lower nucleotide sequences of mRNA and miRNA, respectively. The bold type indicates the nucleotide of noncanonical pairs U-G; A-C.

It should be noted that miRNA binding sites with mRNAs of target genes had the same $\Delta \mathrm{G}(\mathrm{kJ} / \mathrm{mole})$ and $\Delta \mathrm{G} / \Delta \mathrm{Gm}(\%)$ values for each miRNA: for tae-mir408-3p and mRNA of ADARB2, ANO4, FNIP2, TJP3 genes $\triangle \mathrm{G}$ equal $110 \mathrm{~kJ} / \mathrm{mole}$, $\Delta \mathrm{G} / \Delta \mathrm{Gm}$ equal $93 \%$; for zma-miR529 and mRNA of
ATP6VOA4, CHD2, MAP7, MLL, PDE4B genes $\triangle \mathrm{G}$ equal $106 \mathrm{~kJ} / \mathrm{mole}, \Delta \mathrm{G} / \Delta \mathrm{Gm}$ equal $94 \%$; for zma-miR482-3p and mRNA of ATP2A3, CSF1, LARP1, LMBRD2, PTER, STK32A, TMCO5A, TTC25 genes $\triangle \mathrm{G}$ equal $-96 \mathrm{~kJ} / \mathrm{mole}$, $\Delta \mathrm{G} / \Delta \mathrm{Gm}$ equal $92 \%$.

\section{Conclusion}

Thus, the consumption of wheat and maize in food not only provides the human body with energy, but also their miRNAs are involved in the regulation of gene expression, affecting human metabolism. The miRNAs significantly affect many genes, so plant miRNAs can be regulators of human gene expression.

\section{Acknowledgment}

The work was carried out with the financial support of the Ministry of Education and Science of the Republic of Kazakhstan within the framework of the grant №AP05132460.

\section{References}

[1] A. K. Rakhmetullina, A. T. Ivashchenko, "Rice miRNAs are potential regulators of human genes expression," NEWS of the National academy of sciences of the Republic of Kazakhstan, vol. 5, pp. 24-31, 2019. DOI: $10.32014 / 2019.2519-1629.44$

[2] A. Ivashchenko, O. Berillo, A. Pyrkova, R. Niyazova, S. Atambayeva, "MiR-3960 binding sites with mRNA of human genes," Bioinformation, vol. 10, pp. 423-427, 2014. DOI: 10.6026/97320630010423

[3] A. Garg, U. Heinemann, "A novel form of RNA double helix based on G.U and C.A+ wobble base pairing," RNA, vol. 24, pp.209-218, 2018. DOI: $10.1261 /$ rna.064048.117

[4] Q. Gong, J. Xie, Y. Li, Y. Liu, G. Su, "Enhanced ROBO4 is mediated by up-regulation of HIF- $1 \alpha / \mathrm{SP} 1$ or reduction in $\mathrm{miR}-125 \mathrm{~b}-5 \mathrm{p} / \mathrm{miR}$ 146a-5p in diabetic retinopathy," J Cell Mol Med., vol. 23, pp. 47234737, 2019. DOI: $10.1111 / \mathrm{jcmm} .14369$

[5] R. M. de Silva, B. Pupin, T.T. Bhattacharjee, M. A. Vamondes Kulcsar, R. Chammas, R. de Azevedo Canevari, "ATR-FTIR spectroscopy and CDKN1C gene expression in the prediction of lymph nodes metastases in papillary thyroid carcinoma," Spectrochim Acta A Mol Biomol Spectrosc., vol. 228, pp. 117693, 2020. DOI: 10.1016/j.saa.2019.117693

[6] X. Liu, H. Mai, H. Jiang, Z. Xing, D. Peng, Y. Kong, C. Zhu, Y. Chen, "FAM168A participates in the development of chronic myeloid leukemia via BCR-ABL1/AKT1/NFkB pathway," BMC Cancer, vol. 19, pp. 679, 2019. DOI: 10.1186/s12885-019-5898-4

[7] M. Men, J. Wu, Y. Zhao, X. Xing, F. Jiang, R. Zheng, J. D. Li, "Genotypic and phenotypic spectra of FGFR1, FGF8, and FGF17 mutations in a Chinese cohort with idiopathic hypogonadotropic hypogonadism," Fertil Steril., vol. 113, pp. 158-166, 2020. DOI:10.1016/j.fertnstert.2019.08.069

[8] A. L. Coveler, A. H. Ko, D. V. Catenacci, D. Von Hoff, C. Becerra, N.C. Whiting, J. Yang, B. Wolpin, "A phase 1 clinical trial of ASG$5 \mathrm{ME}$, a novel drug-antibody conjugate targeting SLC44A4, in patients with advanced pancreatic and gastric cancers," Invest New Drugs, vol. 34, pp. 319-28, 2016. DOI: 10.1007/s10637-016-0343-X

[9] E. Izquierdo-Torres, A. Hernández-Oliveras, I. Meneses-Morales, G. Rodríguez, G. Fuentes-García, A. Zarain-Herzberg, "Resveratrol upregulates ATP2A3 gene expression in breast cancer cell lines through epigenetic mechanisms," Int J Biochem Cell Biol., vol. 113, pp. 37-47, 2019. DOI: 10.1016/j.biocel.2019.05.020

[10] J. Han, G. Zhan, X. Ma, Q. Dong, H. Zhang, Y. Wang, J. Cui, "CircRNA circ-BANP-mediated miR-503/LARP1 signaling contributes to lung cancer progression," Biochem Biophys Res Commun., vol. 503, pp. 2429-2435. DOI: 10.1016/j.bbrc.2018.06.172 